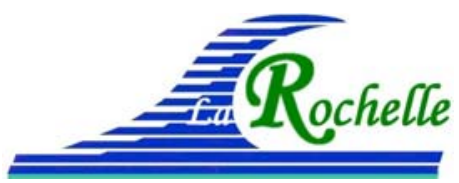

XVèmes Journées Nationales Génie Côtier - Génie Civil

La Rochelle, 29 au 31 mai 2018

DOI:10.5150/jngcgc.2018.069 (C) Editions Paralia CFL

disponible en ligne - http://www.paralia.fr - available online

\title{
Méthode pluridisciplinaire de caractérisation physique des systèmes dunaires côtiers
}

\author{
Vivien BAUDOUIN 1, Manuel GARCIN ${ }^{2}$, Jacques DEPARIS ${ }^{2}$, \\ Emmanuelle PLAT ${ }^{1}$, Adnand BITRI ${ }^{2}$, Cécile LE GUERN ${ }^{1}$, \\ Gwenaëlle BODERE ${ }^{1}$, Gildas NOURY ${ }^{2}$, Angélie PORTAL ${ }^{2}$, \\ Christophe ROLLIER ${ }^{3}$, Loïc GOUGUET ${ }^{4}$, Rémy ARTHUIS 5
}

1. BRGM Direction régionale des Pays de la Loire, 1 rue des Saumonières, BP 92342, 44323 Nantes cedex 3, France.v.baudouin@brgm.fr

2. BRGM Centre Scientifique et Technique, 3 avenue Claude-Guillemin, BP 36009 , 45060 Orléans Cedex 02, France.m.garcin@brgm.fr

3. ONF Agence Travaux, 15 Boulevard Léon Bureau, 44000 Nantes, France. christophe.rollier@onf.fr

4. ONF Département des Risques Naturels, 15 Boulevard Léon Bureau, 44000 Nantes, France.loic.gouguet@onf.fr

5. INRAP Centre de recherches archéologiques de Carquefou, 4 rue du Tertre, 44477 Carquefou cedex, France.remy.arthuis@inrap.fr

\section{Résumé :}

Les cordons dunaires participent aux fermetures de vastes zones basses littorales (marais maritimes, polders, ...), à l'instar des digues. Or ces systèmes littoraux sont encore peu étudiés en termes de structure et de propriétés géomécanique, alors que cette connaissance est nécessaire pour anticiper les risques d'érosion. Une méthodologie de caractérisation physique a été développée et testée sur le littoral du Pays de Monts (Vendée). Quatre sites domaniaux sélectionnés pour leur complémentarité ont été investigués afin de tester des méthodes géophysiques, couplées à des mesures géotechniques in situ et à des analyses de sols. Les premiers résultats apparaissent prometteurs pour la caractérisation des systèmes littoraux sableux, dans une perspective de changement climatique et de hausse du niveau marin.

Mots-clés : Dune, Géophysique, Sédimentologie, Géotechnique, Hydrogéologie, Méthode, Aménagement, Risque, Marais maritime, Changement climatique, Prospective littorale.

\section{Introduction}

Les dunes littorales assurent un ensemble de fonctions variées dans les domaines des risques naturels, de la ressource en eau, de l'aménagement du territoire, des enjeux environnementaux, paysagers et touristiques. En terme de risque, elles contribuent à la résilience du littoral face à l'érosion côtière qui affecte un pourcentage élevé des côtes atlantiques européennes et plus particulièrement françaises. Elles assurent une 


\section{Thème 6 - Gestion durable des zones littorales et estuariennes}

protection efficace contre les submersions marines en jouant un rôle de "digues naturelles" lors des tempêtes. La question de leur évolution dans le cadre du changement climatique et de la remontée du niveau marin associée nécessite une attention particulière.

Le projet SIBLES vise à améliorer la connaissance des paramètres influents le comportement des systèmes dunaires et à développer une méthodologie, reproductible et économiquement accessible, de caractérisation géomorphologique $3 \mathrm{D}$ des cordons dunaires. Une approche multiméthode est proposée dans cette étude, combinant géophysique (sismique, électrique et radar géologique) et sondages géotechniques.

\section{Contexte géologique}

Le site du Pays-de-Monts (Vendée, France) comprend un système dunaire complexe en interface avec un marais rétro-littoral. Depuis 2010, il bénéficie, de suivis du trait de côte et des nappes, conduits dans le cadre de l'Observatoire du Littoral du Pays de Monts (LE GUERN et al., 2014). Il existe également un corpus de données significatif (sondages géotechniques et géologiques) sur ce secteur, découpé en zones d'études plus restreintes, représentant des contextes hydro-morpho-géologiques distincts du Pays-deMonts.

Le substratum induré du Pays de Monts est principalement constitué par des dépôts éocènes du Lutécien formé de grès, calcaires dolomitiques et de marnes blanches (figure 1). Les sédiments meubles sont principalement constitués par des dépôts fluviomarins postérieurs de la transgression de l'Holocène. Ces derniers ("flandriens"), d'une dizaine de mètres d'épaisseur, sont composés d'argiles plus ou moins sableuses à horizons tourbeux (le "bri") et forment le Marais de Monts. Ces dépôts flandriens reposent indifféremment sur les formations antérieures en les masquant. Sur la frange littorale, le complexe dunaire varie lattéralement avec (a) des cordons dunaires bordiers, (b) un ensemble de dunes paraboliques digitées avec des espaces interdunaires assez étendus et (c) des dunes paraboliques chevauchantes de plus de $20 \mathrm{~m}$ de hauteur (GARCIN et al., 2017). Ces dernières, dont la mobilité très récente est attestée jusqu'au XIXème siècle, ont migré progressivement vers l'est et constituent aujourd'hui la bordure du Marais de Monts.

Au-delà de la zone de fermeture du prisme sableux des plages, les sédiments marins actuels sont peu abondants et cantonnés dans de petites dépressions sous-marines correspondant principalement à des paléo-chenaux (BALTZER et al., 2014). L'imbrication du système plage-dune avec le marais maritime est mal connue, particulièrement la géométrie du passage latéral de faciès entre, le prisme sableux marin actuel, et la zone abritée qui favorise la sédimentation d'argiles et de matières organiques. De même, les caractéristiques (géométrie, lithologie) et la localisation du substratum sous le système dunaire et le marais restent mal connues. 


\section{XVèmes Journées Nationales Génie Côtier - Génie Civil \\ La Rochelle, 29 au 31 mai 2018}

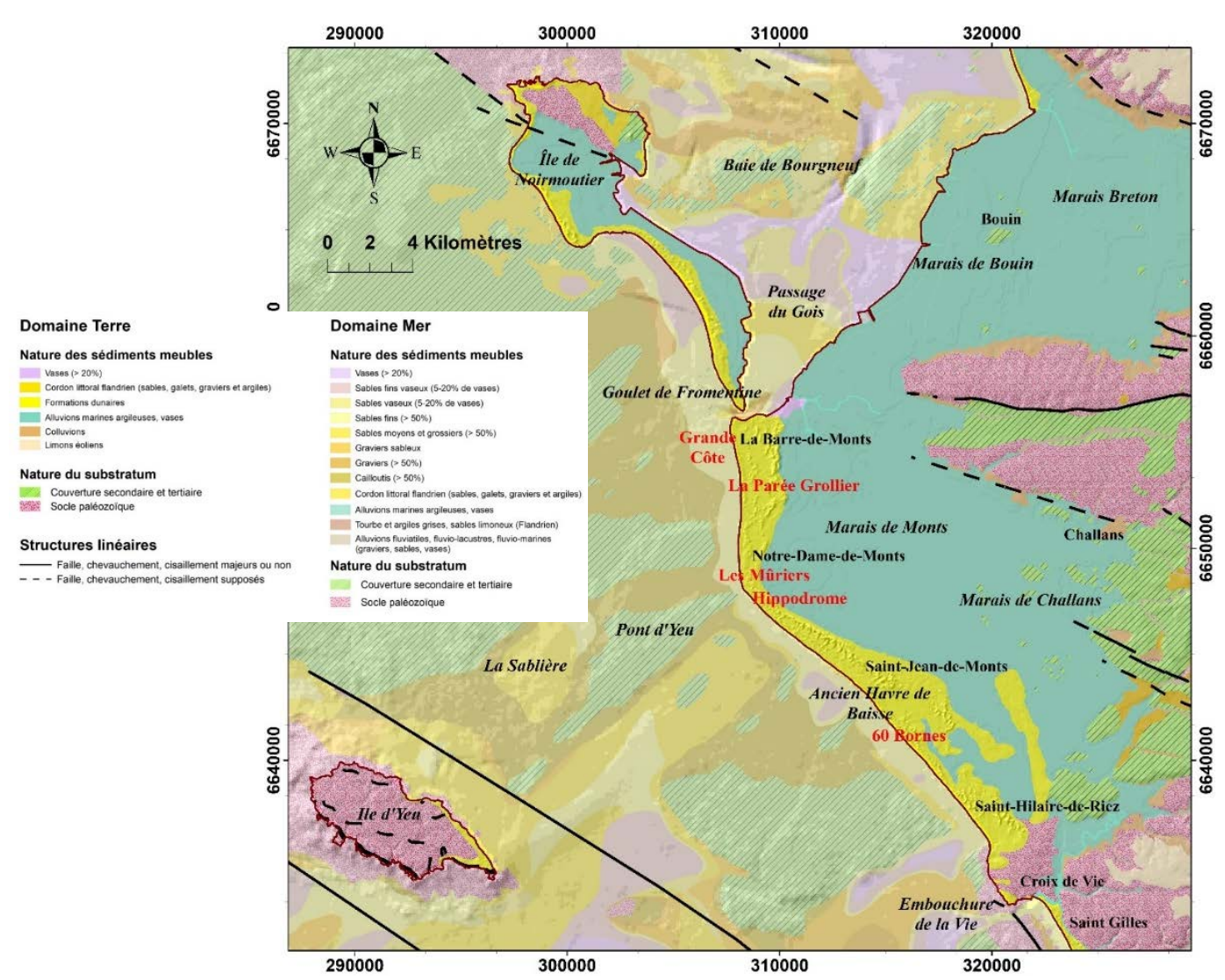

Figure 1. Carte sédimento-géologique synthétique du littoral des Pays de la Loire à 1/250 000 (modifiée d'après THINON et al., 2013)

et choix des sites investigués (en rouge).

\section{Méthodologie}

L'approche méthodologique développée ici consiste à :

a) Identifier une zone d'étude représentative du secteur du Pays-de-Monts, présentant des données géologiques et géotechniques significatives (calage des résultats géophysiques et interpolation globale à l'ensemble de la zone).

b) Réaliser une synthèse de l'histoire géologique récente du secteur ausculté (Quaternaire), afin de déterminer les modalités de mise en place des corps sédimentaires, leurs relations géométriques et leurs natures lithologiques.

c) Mettre en œuvre une campagne d'acquisition de données géophysiques (compaction, résistance et réflexion radar) et géotechniques.

d) Construire une base de données (géophysiques, géotechniques et géologiques) en vue d'établir un modèle 3D des caractéristiques physiques et hydrodynamiques des dunes.

e) Tester des modèles géotechniques conventionnels pour appréhender la résistance à l'érosion des dunes. 


\section{Thème 6 - Gestion durable des zones littorales et estuariennes}

\section{Acquisitions des données}

\subsection{Synthèse des données existantes}

Afin de caractériser les formations meubles superficielles (jusqu'à 15-20 m de prof.) sur l'ensemble du site des Pays de Monts, une synthèse des données de sondages existantes a été réalisée auprès des collectivités et des bureaux d'études locaux, et également à partir de la bibliographie (données de thèses et publications). La base de données ainsi créée contient à ce jour plus de 700 sondages, vérifiés et relocalisés avec précision et dont les données géologiques et géotechniques ont été informatisées (tableau 1).

La répartition spatiale hétérogène des données de sondages (figure 2) ne permet pas une modélisation équilibrée sur l'ensemble du système dunaire des Pays de Monts. La forêt domaniale (ONF) est particulièrement dépourvue de sondages, en dehors de quelques sites particulièrement bien étudiés (Grande Côte, Parée Grollier, Mûriers-Hippodrome et 60 Bornes). En revanche, pour les secteurs bien couverts, la qualité et la diversité des données collectées (mesures géotechniques, analyses granulométriques et reconnaissances de faciès lithologiques) permet d'envisager l'ébauche d'une modalisation géologique et géotechnique. Ces données constituent également un préalable important dans le choix des sites tests pour les investigations géophysiques conduites dans le cadre de ce projet (figure 2).

\subsection{Méthodes géophysiques}

En complément, des acquisitions géophysiques non destructives (figure 2 et tableau 1), ont été mises en œuvre.

\subsubsection{Géoradar}

Un radar ProEx de Mala Geoscience (antennes $500 \mathrm{MHz}$ ) a été utilisé pour effectuer les mesures selon des transects crossshore et longshore (figure 2). Le traitement des données géoradar a été réalisé avec le logiciel ReflexW (Sandmeier). Les enregistrements n'ont été réalisés qu'en dehors de la zone d'estran (trop conductrice). Le milieu étudié au-dessus de la nappe est très résistant électriquement ce qui le rend adapté aux acquisitions géoradar.

\subsubsection{Méthode électrique (Ohmmapper)}

L'acquisition des profils électriques a été réalisée à l'aide d'un dispositif OhmMapper (Geometrics, USA) qui, grâce à un système d'électrodes capacitives, permet de mesurer, en continu, la résistivité électrique en tractant le dispositif au sol. La distance interélectrode de $5 \mathrm{~m}$, selon un dispositif dipôle-dipôle, a permis d'assurer une pour une profondeur d'investigation d'environ $5 \mathrm{~m}$. 


\section{XVèmes Journées Nationales Génie Côtier - Génie Civil \\ La Rochelle, 29 au 31 mai 2018}

\subsubsection{Sismique}

L'acquisition des profils sismiques a été réalisée à l'aide d'une centrale d'acquisition sismique Stratavizor 48 traces (Geometrics, USA) connectée à une flûte de 24 traces (géophones), régulièrement espacées de 2 mètres, tractée par un Quad (prêté par l'ONF). Les capteurs utilisés étaient des géophones tractables de fréquence de résonance $10 \mathrm{~Hz}$. Une source sismique de type marteau $(10 \mathrm{~kg})$ a été utilisée. L'espacement entre deux tirs consécutifs était de $10 \mathrm{~m}$.

\subsection{Acquisitions complémentaires}

\subsubsection{Sondages carottés et tests en laboratoire}

Deux sondages carottés ont été réalisés en complément des sondages existants sur les autres sites. Ils ont été équipés de piézomètres et de sondes afin d'obtenir des mesures en continu du niveau de l'eau (pas de $15 \mathrm{mn}$ ). Les enregistrements obtenus couvrent la période du 12/01/2017 au 07/12/2017. Deux autres ouvrages existants sur le site de 60 Bornes ont également été équipés de sondes. A partir des échantillons de deux sondages réalisés sur le site de la Parée Grollier, 6 analyses granulométriques ont été effectuées, ainsi que de 2 tests de densité de sols.

\subsubsection{Sondages géotechniques (CPT Cone Penetration Test)}

Le CPT est un test de pénétration statique qui offre l'avantage d'être un mode d'investigation particulièrement bien adapté aux milieux littoraux sableux. Il permet d'enregistrer 3 paramètres : la résistance apparente de pointe, la pression interstitielle et de frottement latéral (friction). Outre l'interprétation de ces paramètres géomécaniques, la nature des terrains traversés peut être déterminée à partir de la classification de Robertson (1986). Ces sondages ont été réalisés notamment en vue de caler les investigations géophysiques sur le site de la Parée Grollier.

\subsubsection{Datations radiocarbone}

Les deux sondages carottés de la Parée Grollier n'ont pas présenté d'horizons organiques suffisants pour mesurer l'activité radiocarbone ${ }^{14} \mathrm{C}$. Les prélèvements ont été réalisés à partir d'une sélection dans les carottes de sondages provenant de travaux de reconnaissances géotechniques (pour le raccordement électrique du futur Parc Eolien Yeu-Noirmoutier).

Huit prélèvements ont été analysés, ils concernent les 3 sites suivants : Grande Côte, Mûriers et Hippodrome. 


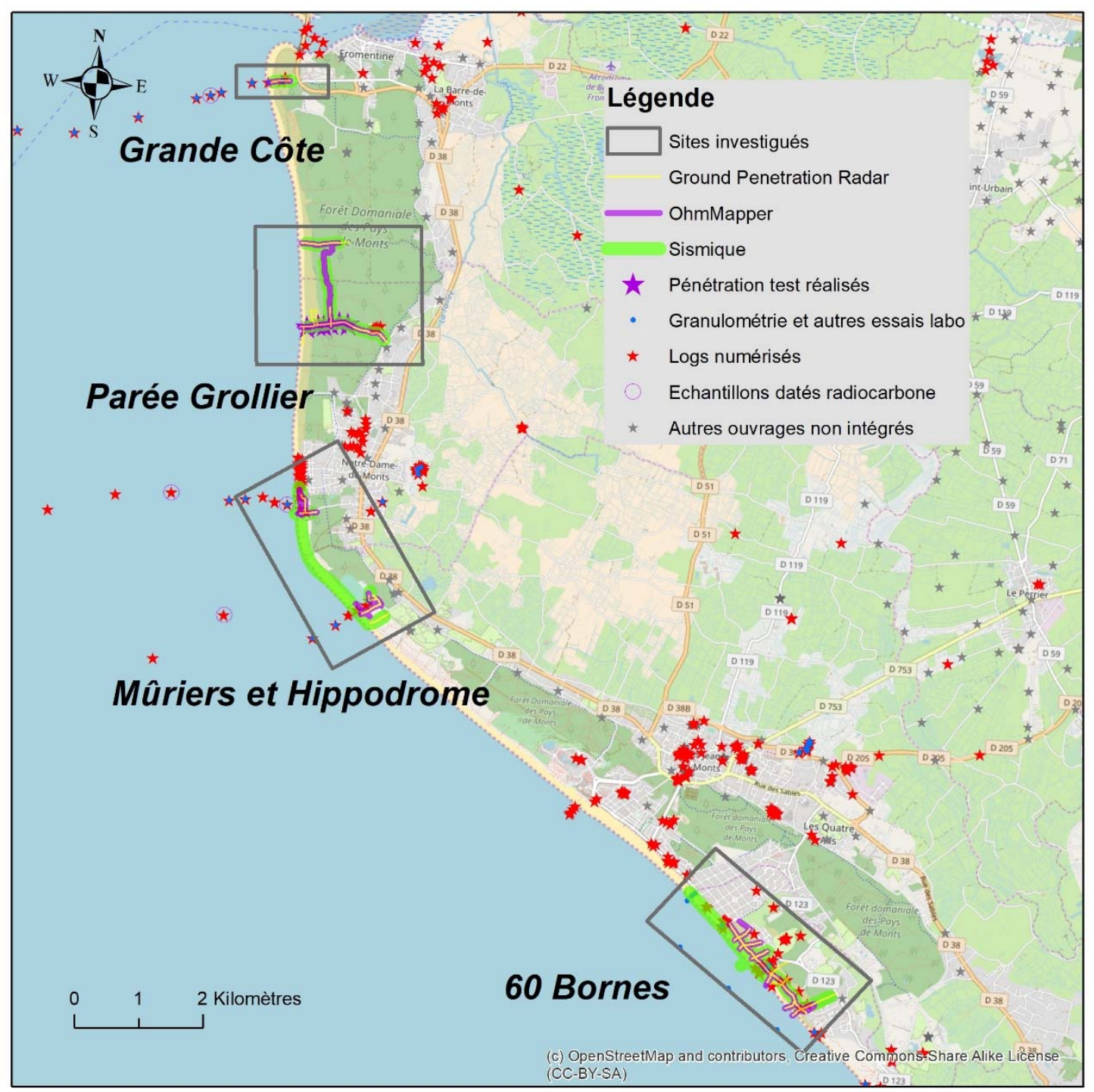

Figure 2. Localisation des données acquises et sélectionnées sur le littoral du Pays de Monts.

Les données acquises ont été intégrées à la base de données réalisée. Elle consultable sous ArcGIS et peut être visualisée en 3D avec l'outil GDM pour ArcGIS (possibilité d'interroger des modèles géologiques 3D à partir d'ArcGIS). 


\section{XVèmes Journées Nationales Génie Côtier - Génie Civil \\ La Rochelle, 29 au 31 mai 2018}

Tableau 1. Synthèse des données acquises.

\begin{tabular}{|c|c|c|c|c|c|c|}
\hline & & \multicolumn{3}{|c|}{ Inventaire, acquisition et collecte } & \multicolumn{2}{|c|}{$\begin{array}{c}\text { Informatisation des données des } \\
\text { sondages }\end{array}$} \\
\hline & Détail & $\begin{array}{c}\text { Campagne } \\
\text { SIBLES }\end{array}$ & $\begin{array}{l}\text { Collecte } \\
\text { spécifique }\end{array}$ & $\begin{array}{c}\text { Existants } \\
\text { (valorisation } \\
\text { BSS) }\end{array}$ & $\begin{array}{l}\text { Nombre de } \\
\text { sondages }\end{array}$ & $\begin{array}{c}\text { Données } \\
\text { (echantillons, } \\
\text { passes } \\
\text { enregistrées) }\end{array}$ \\
\hline Sondages compilés & Tous & & 285 & 850 & 1135 & 1 \\
\hline dont & Carottage & 2 & 27 & 28 & 57 & l \\
\hline \multirow{2}{*}{$\begin{array}{l}\text { Mesures en } \\
\text { laboratoire }\end{array}$} & Granulométrie, essais de sol & 6 & \multicolumn{2}{|r|}{49} & 55 & 67 \\
\hline & Datations & 8 & 9 & 0 & 17 & 60 \\
\hline \multirow{4}{*}{ Mesures in situ } & Pressiométrie & 0 & 123 & 69 & 192 & 1036 \\
\hline & Pénétration test (CPT) & 6 & 32 & 0 & 38 & 1 \\
\hline & \begin{tabular}{|l|} 
Piézométrie \\
\end{tabular} & 2 & 1 & 2 & 5 & 1 \\
\hline & Descriptions lithologiques & 2 & 27 & 551 & 580 & 3150 \\
\hline & \begin{tabular}{|c|} 
Méthode \\
\end{tabular} & Total (m) & Grande Côte & Parée Grollier & Pont d'Yeu & 60 Bornes \\
\hline \multirow{3}{*}{ Profils géophysique } & Radar GPRS & 10007 & \begin{tabular}{|l|}
301 \\
\end{tabular} & 3361 & 1670 & 4676 \\
\hline & Electrique (OhmMapper) & 10460 & 349 & 4122 & 2082 & 3907 \\
\hline & Sismique & 10282 & 277 & 2732 & 3246 & 4027 \\
\hline
\end{tabular}

\section{Résultats et discussion}

\subsection{Apports par méthode}

Sur le profil W-E de la Parée Grollier la paléosurface sur lequel repose les dépôts holocènes se distingue nettement avec la méthode sismique (figure 3)

En corrélant les informations géologiques à notre disposition avec le profil sismique, trois horizons de vitesses sismiques se détachent :

a) Une couche superficielle caractérisée par des vitesses sismiques comprises entre 100 et $300 \mathrm{~m} / \mathrm{s}$. Son épaisseur est variable le long des profils et peut atteindre $10 \mathrm{~m}$ de profondeur au Pm (point métrique) 1350 du profil. De telles vitesses sont caractéristiques des terrains décompactés (remblais récents) ou des terrains sableux et argileux. Une légère diminution des vitesses est observée à partir du Pm $950 \mathrm{du}$ profil ce qui pourrait être interprétée comme la zone de transition avec le "bri".

b) Une deuxième unité caractérisée par des vitesses sismiques comprises entre 350 et $600 \mathrm{~m} / \mathrm{s}$, vitesses caractéristiques des terrains peu compactés. Dans le cas présent elles correspondraient aux vitesses des marnes blanches éocènes ou de niveaux altérés reconnus en sondage. Entre les Pm 500-600 m de cette unité atteint une profondeur de l'ordre de $23 \mathrm{~m}$ avant d'atteindre toit de la couche des calcaires gréseux, plus induré.

c) Cette troisième unité discontinue, dont les vitesses sismiques sont supérieures à $650 \mathrm{~m} / \mathrm{s}$ laisserait supposer une succession de grabens et horsts de direction NNWSSE, témoin d'une activité tectonique importante au cours de l'Eocène (BORNE, 1987).

La sismique permet d'ausculter sur de longues distances (plurikilométriques) l'interface entre les sédiments meubles et le toit du substratum induré. En revanche, le passage latéral d'un système alluvionnaire littoral sableux à un environnement de dépôt abrité (argiles, argiles sableuses, vases, tourbes) est moins perceptible. 


\section{Thème 6 - Gestion durable des zones littorales et estuariennes}
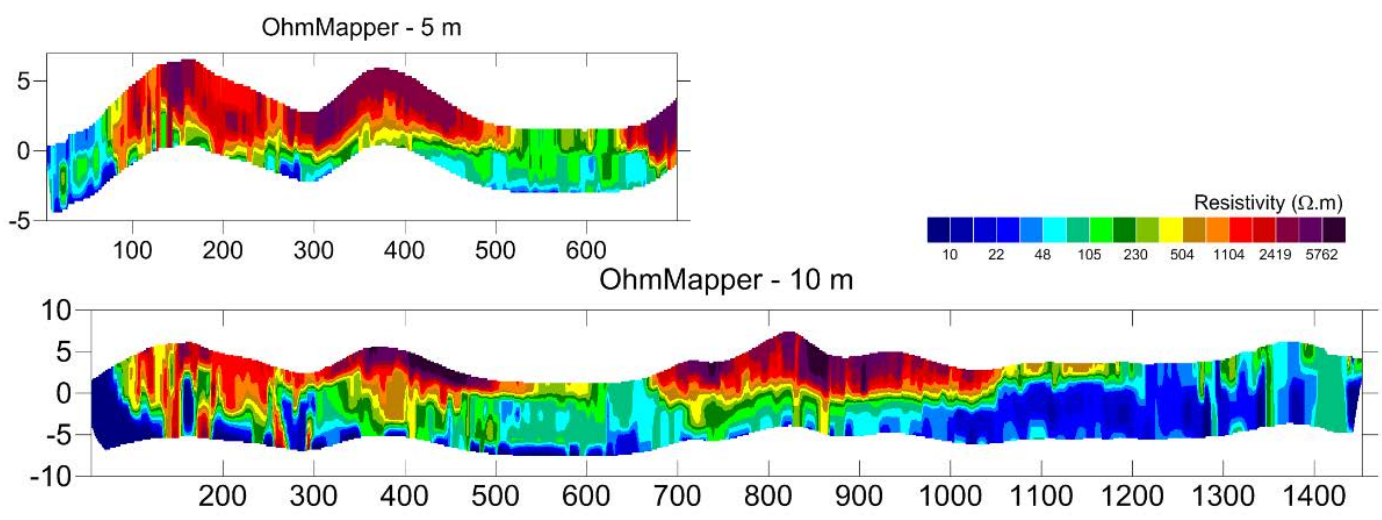

Seismic
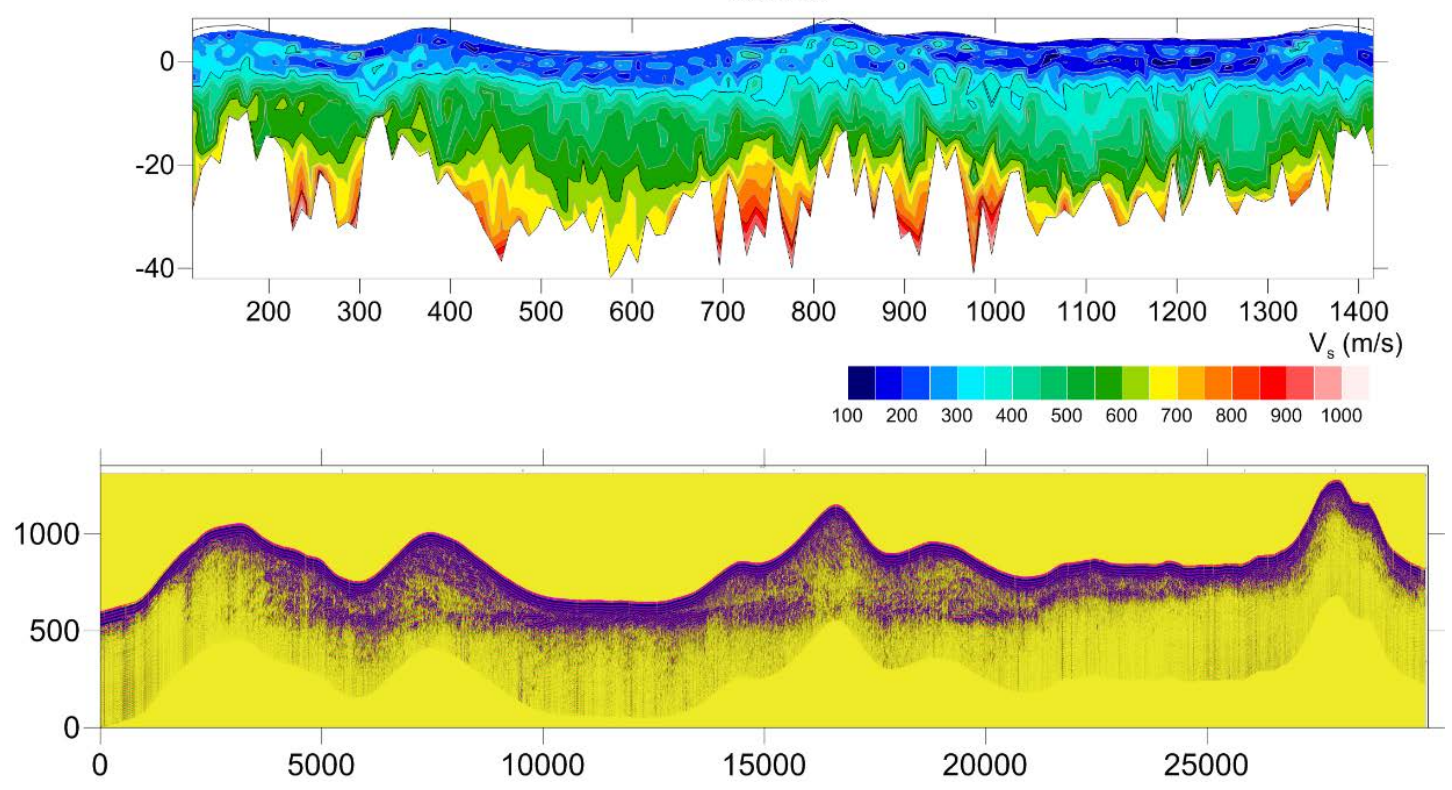

Figure 3. Profils Ouest-Est de la Parée Grollier (Notre Dame de Monts), électriques

(Ohmapper) avec deux niveaux de résolution (5 et $10 \mathrm{~m}$ ), sismique et radar (GPR).

Avec la méthode électrique, les coupes de résistivité inversées font ressortir assez nettement les sables dunaires (très résistants) et une forme d'enracinement, dans une matrice plus conductrice, largement conditionnée par la nappe d'eau douce puis par le front d'eau saumâtre et/ou le biseau salé, que des profils de conductivité, associés à une piézométrie, confirment. Les zones les plus conductrices observées (entre 500 et $650 \mathrm{~m}$ et 1100 et $1400 \mathrm{~m}$ sur la figure 3) peuvent être interprétées comme des milieux plus humides, argileux et/ou organiques.

Enfin, le géoradar (figure $3 \mathrm{du}$ bas) montre des surfaces obliques et parallèles aux versants abrités et illustre la mobilité des dunes par accumulation successive. D'autres surfaces inverses, inclinées vers l'océan, pourraient être interprétées comme d'anciens hauts de plages, témoignant d'un système en accrétion sur cette portion du littoral, ou 


\section{XVèmes Journées Nationales Génie Côtier - Génie Civil \\ La Rochelle, 29 au 31 mai 2018}

bien encore des pieds de dunes et cordons conservés. Des surfaces subhorizontales assez nettes sont également observées à la base des acquisitions, en lien avec le niveau de la nappe et le mode de piégeage et de sédimentation des sables.

Par ailleurs, un travail d'analyse et synthèse des données granulométriques acquises est en cours, afin d'établir les classes granulométriques des corps sédimentaires au sein des dunes et alluvions marines.

\subsection{Complémentarité des méthodes et apports de la pluridisciplinarité}

Les premiers essais d'interprétation croisée ont été réalisés sur le site de la Parée Grollier et permettent à ce stade du projet de tester et consolider la démarche envisagée par une mise en perspective des trois méthodes géophysiques expérimentées (figure 4).

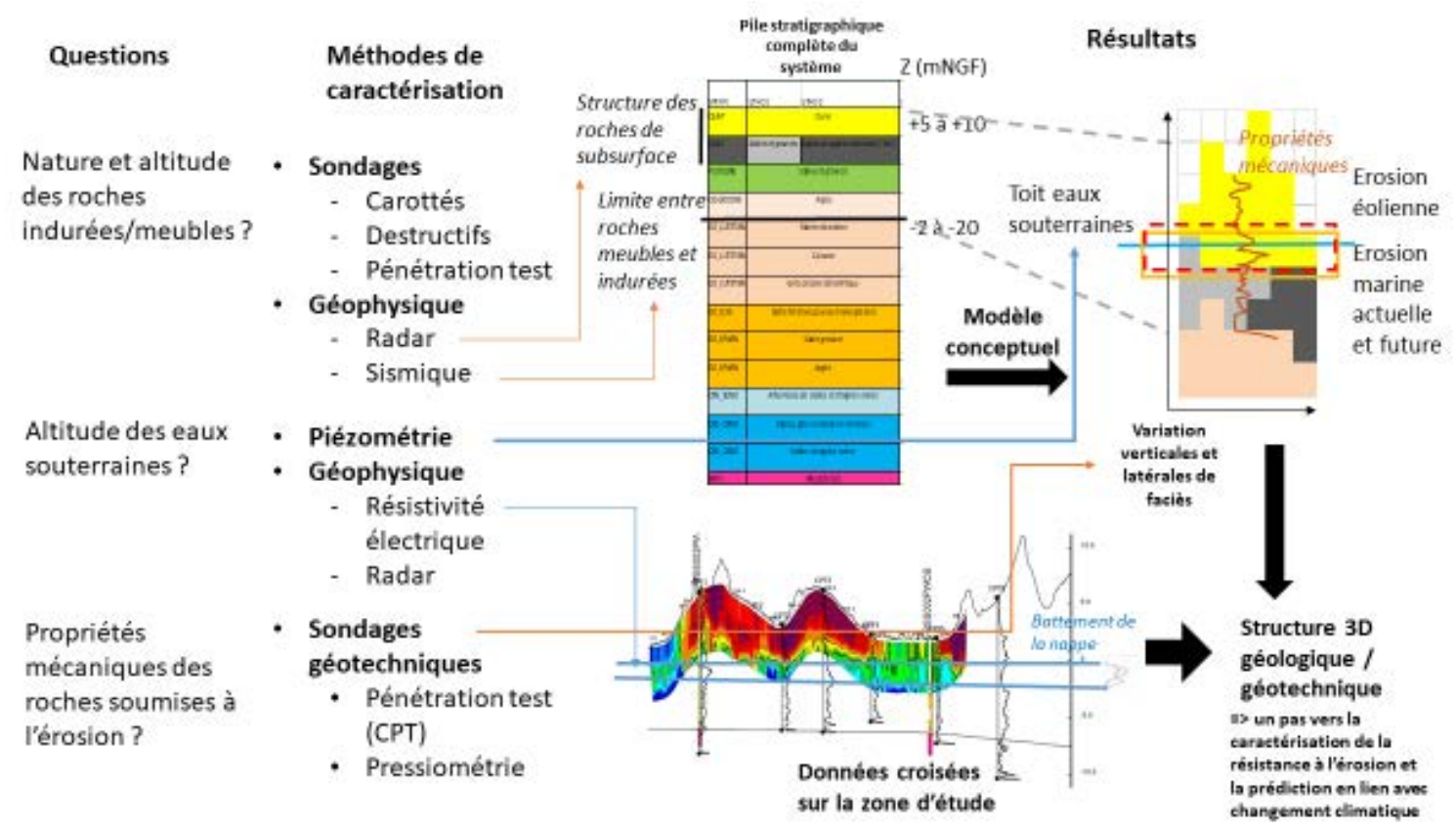

Figure 4. Méthodologie et modèle conceptuel de caractérisation des structures et propriétés géomécaniques associées des littoraux sableux.

A terme il pourrait alimenter des modèles prédictifs, notamment en observant les conséquences de la hausse du niveau marin sur la tranche verticale soumise à l'érosion du système plage-dune.

\section{Conclusions}

La confrontation pluridisciplinaire de ces résultats permet de déterminer différents ensembles homogènes en termes géomorphologique, géomécanique et hydrogéologique Même si l'interprétation est encore à ses débuts, elle permet déjà d'appréhender l'hétérogénéité du système dunaire en lien avec les modalités de sa mise en place. Les 
premiers résultats montrent la pertinence à poursuivre les étapes de la méthode développée pour comprendre et anticiper l'évolution du système dunaire. Cette méthode offre également de nouvelles perspectives pour la gestion environnementale durable des systèmes côtiers dunaires.

\section{Références bibliographiques}

BALTZER A., MAANAN M., ROLLO N., DURAND A. (2014). Approche géophysique de la couverture sédimentaire au large des Pays de Monts. Journées Nationales Génie Côtier - Génie Civil, Dunkerque, pp 539-546. https://doi.org/10.5150/jngcgc.2014.059

BORNE V. (1987). Le bassin paléogène de Challans-Noirmoutier. Thèse Université de Nantes. 285 p.

GARCIN M. et al. (2017). Unpublished

LE GUERN C., BAUDOUIN V., ROBIN M., FATTAL P., CONIL P., JUIGNER M., DEBAINE F., MAANAN M., ROLLIER C., GOUGUET L., BOUCHET F., MAGNE J, ROBIN J.G. (2014). Un SIG pour appuyer la gestion durable du littoral : exemple de l'érosion en Pays de Monts (Vendée, France). Journées Nationales Génie Côtier Génie Civil, Dunkerque, pp 817-824. https://doi.org/10.5150/jngcgc.2014.090

THINON I., BAUDOUIN V., PAQUET F., CONIL P., BECHENNEC F., LE BAYON B. (2013). Cartographie géologique harmonisée du littoral des Pays de la Loire. BRGM RP-62383-FR, 82 p. 